\title{
Eradication of $\boldsymbol{H}$. pylori infection: the challenge is on if standard therapy fails
}

\author{
Ulrich R.M. Bohr and Peter Malfertheiner
}

\begin{abstract}
The recommended standard triple therapy for Helicobacter pylori infection, consisting of a proton pump inhibitor, clarithromycin and amoxicillin or metronidazole, can reach eradication rates in over $90 \%$. However, in recent years resistance to antibiotics has increased and eradication rates have declined. Approximately one in five patients need a second-line therapy because eradication therapy fails. Second-line treatment with a bismuth-based quadruple therapy leads to satisfactory eradication rates, but bismuth is not available in many countries. Modern second- and third-line treatments can only be successful if they are adapted to the current resistance situation and they need to evolve continuously. Moreover, pharmacodynamic effects due to polymorphisms of the cytochrome P450 system are important. Because therapy adherence is significantly associated with therapy success, modern regimens if possible should be easy to take and well tolerated. In recent years, various novel salvage-therapy regimens have been investigated that significantly improve treatment options.
\end{abstract}

Keywords: Helicobacter pylori, triple therapy, proton pump inhibitor, clarithromycin, metronidazole, amoxicillin, bismuth, cytochrome P450

\section{Introduction}

According to the Maastricht III Consensus [Malfertheiner et al. 2007] the recommended first-line therapy for Helicobacter pylori eradication is a proton pump inhibitor (PPI), clarithromycin and amoxicillin or metronidazole, if the primary resistance to clarithromycin in the area is lower than 15-20\%. There is a small advantage in using metronidazole instead of amoxicillin and, therefore, this combination was found to be preferable in areas where the prevalence of metronidazole resistance is lower than $40 \%$. Treatment success of a 14-day treatment may be more effective compared to a 7-day treatment [Ford and Moayyedi, 2003], but a 7-day treatment may be acceptable where local studies have shown high efficacy. When available, bismuthbased quadruple therapies can be used as alternative first-line therapies [Malfertheiner et al. 2007].

If first-line therapy with PPI triple therapy fails, according to the Maastricht III Consensus a second treatment with a bismuth-based quadruple therapy is recommended. If bismuth was previously used or if bismuth is not available, PPI plus amoxicillin or tetracycline and metronidazole is recommended as an alternative. For patients who fail second-line treatment, current guidelines suggest an individually tailored caseby-case approach at the specialist care level based on antimicrobial susceptibility testing [Malfertheiner et al. 2007].

When the Maastricht III guidelines were made in, 2005, only limited data or studies on retreatment if initial therapy failed were available. Therefore, the recommendations had to be given at a rather low level of evidence (2c, based on noncontrolled cohort studies). In recent years, a number of controlled studies increased our knowledge about $H$. pylori retreatment after failure of initial therapy. These new data have to be taken into account if a salvage therapy is necessary. Although current data are not sufficient for general recommendations, there are a number of new aspects that influence therapy decisions and offer new effective treatment options for an increasing number of patients who fail first-line therapy.
Therapeutic Advances in Gastroenterology (2009) 2(1) 59-66 DOI: $10.1177 /$ $1756283 \times 08100352$

(C) The Author(s), 2009. Reprints and permissions: http://www.sagepub.co.uk/ journalsPermissions.nav
Correspondence to: Peter Malfertheiner Department of Gastroenterology, Hepatology and Infectious Diseases, Otto-von-Guericke University Magdeburg, Magdeburg, Germany. peter.malfertheinera med.ovgu.de Ulrich R.M. Bohr Department of Gastroenterology, Hepatology and Infectious Diseases, Otto-vonGuericke University Magdeburg, Magdeburg, Germany 


\section{Why do eradication therapies fail?}

Even with the correct use of drug combinations, infection cannot be eradicated in up to $23 \%$ of patients that receive first-line eradication therapy [Parente et al. 2003]. In patients that receive retreatment, eradication rates further decline. Analysis and elimination of the factors that led to therapy failure in the individual patient is important so as to increase the chance of successful eradication with a subsequent treatment. The key factors that increase the risk of Helicobacter pylori eradication therapy failure are as follows:

- discontinuation of therapy because of adverse effects

- poor adherence to therapy

- pretreatment antibiotic-resistant $H$. pylori

- pharmacodynamic effects.

These will now be discussed.

Adverse events

Side-effects are frequent with $H$. pylori eradication therapy and increase with the number of previous therapies [Gisbert et al. 2008a]. While first line-therapies lead to approximately onequarter to one-third of patients reporting adverse advents, this increases to more than half of the patients who receive third-line therapy [Gisbert et al. 2008a]. This is especially important because side-effects reduce therapy adherence and decrease efficacy of a therapy [Cheon et al. 2006]. Therapy additives, for example probiotics or lactoferrin, can significantly reduce sideeffects and improve eradication rates [Tong et al. 2007; Tursi et al. 2007; Di Mario et al. 2006]. In a meta-analysis that included randomized trials with a total of, 1671 patients, supplementation with probiotics was shown to reduce therapy-related side effects by $15 \%$ and increase eradication rates by almost $10 \%$ [Tong et al. 2007]. Therefore, add-on medications provide an excellent tool to minimize side effects and to improve eradication rates in patients that experienced side-effects with previous unsuccessful $H$. pylori treatments.

\section{Poor adherence to therapy}

Complicated therapy regimens and high pill burden reduce adherence and therapeutic efficacy [Bartlett et al. 2001]. Some of the eradication therapies are rather complex and have a high pill burden. For example, quadruple therapies containing bismuth salts that are currently recommended in second-line therapy can have a pill burden of up to 10 pills per day. Together with a high frequency of side-effects this reduces therapy adherence [Navarro-Jarabo et al. 2007]. In recent years, many studies were conducted to look at improving second-line and salvage therapies by making them simpler, and to reduce pill burden as well as side effects. Several of the alternative therapies used in second-line therapy can reach higher eradication rates than conventional quadruple therapy. Currently, the simplest therapy with the lowest pill burden is a once-daily therapy with only three pills per day [Miehlke et al. 2008]. Despite the low number of pills, this therapy is highly effective and reaches an eradication rate of $77.7 \%$ in pretreated patients with persisting $H$. pylori infection resistant to both metronidazole and clarithromycin.

As well as type of therapy, diagnosis (duodenal ulcer/nonulcer dyspepsia) was demonstrated to be an independent predictor of eradication failure; however, this was not the case in other studies [Chung et al. 2007; Gisbert et al. 1999]. A potential explanation could be that the patient's diagnosis is linked to therapy motivation. While motivation is difficult to measure, it is without doubt that motivation increases therapy adherence. Therefore, after failure of $H$. pylori eradication, the therapy motivation of the patient should be checked, and improved if necessary, before initiating the next eradication attempt.

\section{Pretreatment antibiotic-resistant H. pylori}

Pretreatment antibiotic-resistant $H$. pylori reduces the cure rate of infection by up to $66 \%$ [Realdi et al. 1999]. Metronidazole resistance reduces effectiveness by an average of $37.7 \%$, whereas clarithromycin resistance reduces it by an average of 55\% [Dore et al. 2000]. Resistance increases with the number of unsuccessful treatments. After two eradication failures one can assume that almost all patients carry $H$. pylori strains that are resistant to metronidazole and clarithromycin [Cammarota et al. 2004]. Resistance to quinolone antibiotics can reach up to $31 \%$ [Cammarota et al. 2004]. Resistance to rifabutin, tetracycline and amoxicillin is rare [Cammarota et al. 2004; Heep et al. 2000]. Resistance to clarithromycin is associated with metronidazole resistance in 85-89\% [Heep et al. 2000].

Eradication rates can be increased significantly by susceptibility-based treatments [Lamouliatte 
et al. 2003]; however, in clinical practice susceptibility testing is often not available or is not performed for other reasons. Also, a number of studies showed that empirical second- or thirdline therapies may have excellent therapeutic results; however, it must be kept in mind that for some of the patients who were treated empirically susceptibility testing could have predicted therapy failure. This is nicely shown in patients who receive a second-line PPI-based triple therapy containing levofloxacin. Statistically, salvage therapies containing levofloxacin are highly effective empirical therapies because levofloxacinresistant strains are still rare [Cammarota et al. 2004; Heep et al. 2000]. However, in the small number of patients who are infected with levofloxacin-resistant $H$. pylori, levofloxacin-containing triple therapy is useless because therapy fails in 67-100\% [Perna et al. 2007; Wong et al. 2006].

If empirical second- or third-line therapies are used, treatment history is critically important. An excellent example is the study of Treiber et al. who showed that in patients who failed a standard PPI-based triple therapy containing clarithromycin and metronidazole, second-line quadruple therapy containing tetracycline and metronidazole was successful only in $39 \%$ of the patients. In this study, $90 \%$ of the patients could be cured if the metronidazole was replaced by furazolidone [Treiber et al. 2002].

\section{Pharmacodynamic effects}

PPIs undergo extensive hepatic biotransformation by the cytochrome P450 (CYP) isoenzymes CYP2C19 and CYP3A4 [Furuta et al. 2005]. Genetic polymorphism of CYP2C19, the so-called extensive metabolizer genotype, can significantly increase the activity of CYP2C19 and thus result in a rapid inactivation of PPIs and in consequence reduces acid inhibition. Effective acid inhibition, however, is crucial for successful $H$. pylori eradication because it increases the bioavailability of antibiotics in the gastric mucus by altering gastric volumes and increasing the stability of some antibiotics. The antibiotic effect of amoxicillin on $H$. pylori depends mainly on the time that drug levels are above the minimum inhibitory concentration (MIC), but not on the area under the curve (AUC) or maximum plasma concentration $\left(\mathrm{C}_{\max }\right)$ of the antibiotic [Furuta et al. 2005]. Therefore, a shorter time of sufficient acid suppression due to the extensive metabolizer genotype can have a strong impact on the success of H. pylori eradication therapy. To date, systematic studies that evaluate the impact of the extensive metabolizer genotype on $H$. pylori eradication are rare. Studies from Japan show that patients who fail eradication therapy are often extensive metabolizers. In some studies, up to $100 \%$ of the patients who fail eradication therapy are homozygous or heterozygous for the extensive metabolizer genotype [Togawa et al. 2005; Furuta et al. 2003]. Most likely the combination of a resistant strain of $H$. pylori and extensive metabolizer genotype results in therapy failure in these patients. Adapting the PPI dose and dosing scheme to the metabolization rate in patients with therapy failure or changing to a PPI with a different metabolization pattern are useful strategies to increase eradication rates in patients with CYP2C19 polymorphisms who have already failed previous eradication therapies [Miehlke et al. 2006; Togawa et al. 2005; Furuta et al. 2003].

Interleukin- $1 \beta$ or multidrug resistance (MDR) 1 gene polymorphisms are further factors that may influence eradication rates by pharmacodynamic effects; however, these polymorphisms seem to be less important [Furuta et al. 2007, 2004; Take et al. 2003]. Currently, genotyping of CYP2C19 or other polymorphisms is only a research instrument, but it may become a valuable clinical tool to improve eradication rates in $H$. pylori salvage therapy.

In an expert setting where methods like susceptibility testing and CYP2C19 genotyping are available, eradication therapies can be individually tailored and therapy regimens adjusted to the individual needs of the patient. However, to reach the wide majority of $H$. pylori-infected patients it is important to have treatment strategies that can be done by primary care physicians who usually do not have these specialized methods available. Therefore, for primary care, clear treatment strategies are important that are simple and do not depend on specialized methods. In recent years, various novel salvage-therapy regimens have been evaluated that significantly improve the treatment options.

\section{New developments in bismuth-containing therapies}

Bismuth-based quadruple therapy has proven to be an effective second-line therapy 
[Boixeda et al. 2002; Dore et al. 2002]. Currently, the recommended second-line quadruple therapy is a PPI, together with bismuth, tetracycline and metronidazole; however, other combinations of antibiotics may be effective as well. Recently three quadruple-therapy regimens were compared in a randomized study. All regimens contained bismuth subcitrate and lansoprazole plus two antibiotics. The combination with metronidazole and tetracycline as well as the combination with tetracycline and amoxicillin, with eradication rates of $82.1 \%$ and $81.5 \%$ respectively, were slightly more effective than a third quadruple therapy containing metronidazole and amoxicillin that resulted in an eradication rate of $74.7 \%$ [Uygun et al. 2008]. A cultureguided third-line quadruple regimen including omeprazole, bismuth, doxycycline and amoxicillin was effective in $91 \%$ even in subjects with resistance to multiple antibiotics [Cammarota et al. 2004]. Quadruple therapy with esomeprazole, bismuth, amoxicillin, and levofloxacin was inferior to the recommended second-line quadruple therapy with the combination of PPI, bismuth, tetracycline and metronidazole in a controlled study with an intention-to-treat eradication rate of $56 \%$ versus $90 \%$, respectively [Yee et al. 2007]. In patients who failed PPI-based first-line triple therapy containing clarithromycin and metronidazole, second-line quadruple therapy according to the Maastricht consensus containing metronidazole and tetracycline was ineffective with eradication rates of only $39 \%$. This may be explained by the likelihood of metronidazole resistance in those patients. In consequence, substitution of metronidazole by furazolidone increases the eradication rate to 90\% [Treiber et al. 2002].

Despite good treatment results, bismuth-based quadruple therapy also has disadvantages. Efficacy of quadruple therapies containing bismuth salts are often limited by poor patient compliance due to side-effects, and number of tablets per day [Cheon et al. 2006; Perri et al. 2001]. Moreover, bismuth is not available in all countries. Therefore, PPI-based triple therapies that have less pill burden, are easier to take and are believed to have fewer side-effects have been evaluated as alternative treatment options. There are several randomized head-to-head studies that compare conventional quadruple second-line therapy containing omeprazole, bismuth citrate, tetracycline and metronidazole with PPI-based triple therapy containing a PPI and two antibiotics.
Generally, these studies show that novel secondline triple-therapies have higher eradication rates and are superior with respect to side-effects [Kang et al. 2007; Cheon et al. 2006; Wong et al. 2006; Nista et al. 2003; Perri et al. 2001]; however, there are also individual studies that show the opposite results [Navarro-Jarabo et al. 2007] or have comparable results for both therapy regimens [Kang et al. 2007; Wong et al. 2003]. Good results for PPI-based triple therapies in randomized head-to-head studies were found for the combinations of amoxicillin plus rifabutin [Perri et al. 2001], moxifloxacin plus amoxicillin [Kang et al. 2007; Cheon et al. 2006], levofloxacin plus amoxicillin [Saad et al. 2006; Wong et al. 2006], and rifabutin plus levofloxacin [Wong et al. 2003].

Another option to reduce pill burden and make bismuth-based therapy simpler is to use ranitidine bismuth citrate instead of a PPI and bismuth. A randomized study that compared a 7-day course of second-line therapy with omeprazole, bismuth, tetracycline and metronidazole versus ranitidine bismuth citrate, tetracycline and metronidazole showed a significantly better eradication rate in those patients who received ranitidine bismuth citrate ( $83 \%$ versus $57 \%$, respectively). Surprisingly, the authors stated that adverse effects were infrequent and mild with both regimens [Gisbert et al. 1999]. A second controlled study comparing ranitidine bismuth citrate, tetracycline and metronidazole with a PPI-based triple therapy containing omeprazole, levofloxacin and amoxicillin showed similar results in both groups for cure rates, side-effects and adherence [Gisbert et al. 2007]. Ranitidine bismuth citrate, amoxicillin and tinidazole resulted in comparable eradication rates to a quadruple-therapy regimen [Perri et al. 2003].

\section{New combinations of antibiotics in PPI-based triple therapies that can be used after failure of standard therapy}

\section{PPI, levofloxacin and amoxicillin}

Triple therapy containing a PPI, levofloxacin, and amoxicillin has been proven to be effective as empirical second-line and third-line therapies [Gisbert et al. 2008a, 2008b; Perna et al. 2007; Watanabe et al. 2003]. A treatment for 10 days resulted in eradication rates of $77 \%$ in patients in whom a first treatment with PPI, clarithromycin 
and amoxicillin had failed [Gisbert et al. 2008b]. Therapy duration of 7 days seemed to be inferior to 10-days therapy [Saad et al. 2006]. Eradication therapy with PPI, levofloxacin and amoxicillin is not effective in levofloxacin-resitant $H$. pylori strains [Perna et al. 2007; Wong et al. 2006]; thus, pretreatment testing for levofloxacin resistance is recommended [Wong et al. 2006].

\section{PPI, moxifloxacin and amoxicillin}

Empirical second-line therapies with PPI, moxifloxacin and amoxicillin result in eradication rates of $71.9 \%$ to $75.6 \%$ [Kang et al. 2007; Cheon et al. 2006]. In head-to-head comparison PPI-based triple therapy with moxifloxacin and amoxicillin was at least equal in terms of eradication rate and superior in terms of side-effects to bismuth containing quadruple therapy [Kang et al. 2007; Cheon et al. 2006].

\section{PPI, moxifloxacin and rifabutin}

Currently, PPI-based triple therapy with moxifloxacin and rifabutin is the only once-daily eradication therapy with proven efficacy even in pretreated patient with persistent $H$. pylori infection resistant to both metronidazole and clarithromycin. The regimen resulted in an eradication rate of $77.7 \%$ in the intention-to-treat analysis [Miehlke et al. 2008]. To date, however, there are no head-to-head studies for this regimen. Also, the therapy could be problematic in patients with the extensive metabolizer genotype.

\section{$P P I$, levofloxacin and rifabutin}

Second-line triple therapy with levofloxacin and rifabutin has been shown to be equally effective to quadruple therapy with eradication rates of $91 \%$ for both regimens [Wong et al. 2003].

\section{$P P I$, amoxicillin and rifabutin}

Triple therapy consisting of standard-dose PPI, amoxicillin and rifabutin twice daily for 10 days successfully eradicates $H$. pylori in $72 \%$ of patients (intention-to-treat analysis) [van der Poorten and Katelaris, 2007]. This can be increased to intention-to-treat eradication rates of $90 \%$ with 12 days therapy and three times daily administration of amoxicillin and PPI [Borody et al. 2006], or by increasing the rifabutin daily dosage from 150 to $300 \mathrm{mg}$ [Perri et al. 2001]. Rifabutin $150 \mathrm{mg}$ daily is not recommended [Navarro-Jarabo et al. 2007; Perri et al. 2001]. If PPI-based triple-therapy with amoxicillin and rifabutin is used as a third-line therapy (i.e. after failure of the standard first-line therapy and consecutive quadruple-therapy), efficacy can drop to a cure rate of only 45\% [Gisbert et al. 2006b]; however, there may be regional differences that have to be further evaluated [Miehlke et al. 2006; Gisbert et al. 2003; Perri et al. 2000]

\section{Other interesting antibiotics and novel treat- ment strategies}

Faropenem is the first orally-active carbapenem antibiotic. It has been available under the trade name Farom in Japan since, 1997. In a Japanese study, a second-line therapy with rabeprazole, amoxicillin and faropenem reached an intention-to-treat eradication rate of $91.3 \%$ [Togawa et al. 2005]. Morover, the faropenem-containing treatment regimen was effective despite the fact that all patients in the study were homozygous or heterozygous for the extensive metabolizer genotype.

Other interesting antibiotics for salvage therapy are tinidazole [Nista et al. 2003; Watanabe et al. 2003] and nitrofuran [Ebrahimi-Dariani et al. 2003; Isakov et al. 2002; Treiber et al. 2002]. A new sequential first-line treatment strategy was shown to achieve higher eradication rates compared to the standard regimen [Zullo et al. 2003]. Until now, there have been no studies that evaluated sequential treatments in patients that already failed one or more eradication therapies.

\section{Conclusion}

The authors recommend the following steps in treating patients who have already failed eradication therapy:

- Carefully analyse the individual reasons that led to eradication failure(s).

- Use susceptibility testing whenever possible.

- Do not use antibiotics previously used with potential post-therapeutic resistance.

- Ensure sufficient acid suppression.

- Motivate the patient in order to increase therapy adherence.

\section{Conflict of interest statement}

Peter Malfertheiner receives research grants from AstraZeneca. Speakers bureau with AstraZeneca, Nycomed, Abbott. 


\section{References}

Bartlett, J.A., DeMasi, R., Quinn, J., Moxham, C. and Rousseau, F. (2001) Overview of the effectiveness of triple combination therapy in antiretroviral-naive HIV-1 infected adults, AIDS 15(11): 1369-1377.

Boixeda, D., Bermejo, F., Martín-De-Argila, C., López-Sanromán, A., Defarges, V., HernándezRanz, F. et al. (2002) Efficacy of quadruple therapy with pantoprazole, bismuth, tetracycline and metronidazole as rescue treatment for Helicobacter pylori infection, Aliment Pharmacol Ther 16(8): 1457-1460.

Borody, T.J., Pang, G., Wettstein, A.R., Clancy, R., Herdman, K., Surace, R. et al. (2006) Efficacy and safety of rifabutin-containing 'rescue therapy' for resistant Helicobacter pylori infection, Aliment Pharmacol Ther 23(4): 481-488.

Cammarota, G., Martino, A., Pirozzi, G., Cianci, R., Branca, G., Nista, E.C. et al. (2004) High efficacy of 1 -week doxycycline- and amoxicillin-based quadruple regimen in a culture-guided, third-line treatment approach for Helicobacter pylori infection, Aliment Pharmacol Ther 19(7): 789-795.

Cheon, J.H., Kim, N., Lee, D.H., Kim, J.M., Kim, J.S., Jung, H.C. et al. (2006) Efficacy of moxifloxacin-based triple therapy as second-line treatment for Helicobacter pylori infection, Helicobacter 11(1): 46-51.

Chung, S.J., Lee, D.H., Kim, N., Jung, S.H., Kim, J.W., Hwang, J.H. et al. (2007) Eradication rates of Helicobacter pylori infection with second-line treatment: non-ulcer dyspepsia compared to peptic ulcer disease, Hepatogastroenterology 54(76): 1293-1296.

Di Mario, F., Aragona, G., Dal Bó, N., Cavallaro, L., Marcon, V., Olivieri, P. et al. (2006) Bovine lactoferrin for Helicobacter pylori eradication: an open, randomized, multicentre study, Aliment Pharmacol Ther 23(8): 1235-1240.

Dore, M.P., Leandro, G., Realdi, G., Sepulveda, A.R. and Graham, D.Y. (2000) Effect of pretreatment antibiotic resistance to metronidazole and clarithromycin on outcome of Helicobacter pylori therapy: a meta-analytical approach, Dig Dis Sci 45(1): 68-76.

Dore, M.P., Graham, D.Y., Mele, R., Marras, L., Nieddu, S., Manca, A. et al. (2002) Colloidal bismuth subcitrate-based twice-a-day quadruple therapy as primary or salvage therapy for Helicobacter pylori infection, Am $\mathcal{F}$ Gastroenterol 97(4): 857-860.

Ebrahimi-Dariani, N., Mirmomen, S., MansourGhanaei, F., Noormohammadpoor, P., Sotodehmanesh, R., Haghpanah, B. et al. (2003) The efficacy of furazolidone-based quadruple therapy for eradication of Helicobacter pylori infection in Iranian patients resistant to metronidazole-based quadruple therapy, Med Sci Monit 9(8): 105-108.

Ford, A. and Moayyedi, P. (2003) How can the current strategies for Helicobacter pylori eradication therapy be improved?, Can $\mathcal{F}$ Gastroenterol Suppl. B): 36B-40B.
Furuta, T., Shirai, N., Xiao, F., Takashita, M., Sugimoto, M., Kajimura, M. et al. (2003) High-dose rabeprazole/amoxicillin therapy as the second-line regimen after failure to eradicate $\mathrm{H}$. pylori by triple therapy with the usual doses of a proton pump inhibitor, clarithromycin and amoxicillin,

Hepatogastroenterology 50(54): 2274-2278.

Furuta, T., Shirai, N., Xiao, F., El-Omar, E.M., Rabkin, C.S., Sugimura, H. et al. (2004)

Polymorphism of interleukin-1beta affects the eradication rates of Helicobacter pylori by triple therapy, Clin Gastroenterol Hepatol 2(1): 22-30.

Furuta, T., Shirai, N., Sugimoto, M., Nakamura, A., Hishida, A. and Ishizaki, T (2005) Influence of CYP2C19 pharmacogenetic polymorphism on proton pump inhibitor-based therapies, Drug Metab Pharmacokinet 20(3): 153-167.

Furuta, T., Sugimoto, M., Shirai, N., Matsushita, F., Nakajima, H., Kumagai, J. et al. (2007) Effect of MDR1 C3435T polymorphism on cure rates of Helicobacter pylori infection by triple therapy with lansoprazole, amoxicillin and clarithromycin in relation to CYP 2C19 genotypes and 23S rRNA genotypes of H. pylori, Aliment Pharmacol Ther 26(5): 693-703.

Gisbert, J.P., Gisbert, J.L., Marcos, S., Grávalos, R.G., Carpio, D. and Pajares, J.M. (1999) Seven-day 'rescue' therapy after Helicobacter pylori treatment failure: omeprazole, bismuth, tetracycline and metronidazole vs. ranitidine bismuth citrate, tetracycline and metronidazole, Aliment Pharmacol Ther 13(10): 1311-1316.

Gisbert, J.P., Calvet, X., Bujanda, L., Marcos, S., Gisbert, J.L. and Pajares, J.M. (2003) Rescue therapy with rifabutin after multiple Helicobacter pylori treatment failures, Helicobacter 8(2): 90-94.

Gisbert, J.P., Castro-Fernández, M., Bermejo, F., Pérez-Aisa, A., Ducons, J., Fernández-Bermejo, M. et al. (2006a) Third-line rescue therapy with levofloxacin after two H. pylori treatment failures, $A m \mathcal{F}$ Gastroenterol 101(2): 243-247.

Gisbert, J.P., Gisbert, J.L., Marcos, S., MorenoOtero, R. and Pajares, J.M. (2006b) Third-line rescue therapy with levofloxacin is more effective than rifabutin rescue regimen after two Helicobacter pylori treatment failures, Aliment Pharmacol Ther 24(10): 1469-1474.

Gisbert, J.P., Gisbert, J.L., Marcos, S., MorenoOtero, R. and Pajares, J.M. (2007) Levofloxacin- vs. ranitidine bismuth citrate-containing therapy after H. pylori treatment failure, Helicobacter 12(1): 68-73.

Gisbert, J.P., Gisbert, J.L., Marcos, S., JimenezAlonso, I., Moreno-Otero, R. and Pajares, J.M. (2008a) Empirical rescue therapy after Helicobacter pylori treatment failure: a 10-year single-centre study of 500 patients, Aliment Pharmacol Ther 27(4): 346-354.

Gisbert, J.P., Bermejo, F., Castro-Fernández, M., Pérez-Aisa, A., Fernández-Bermejo, M., Tomas, A. et al. (2008b) Second-line rescue therapy with levofloxacin after $H$. pylori treatment failure: a Spanish 
multicenter study of 300 patients, Am $\mathcal{F}$ Gastroenterol 103(1): 71-76.

Heep, M., Kist, M., Strobel, S., Beck, D. and Lehn, N. (2000) Secondary resistance among 554 isolates of Helicobacter pylori after failure of therapy, Eur $\mathcal{F}$ Clin Microbiol Infect Dis 19(7): 538-541.

Isakov, V., Domareva, I., Koudryavtseva, L., Maev, I. and Ganskaya, Z. (2002) Furazolidone-based triple 'rescue therapy' vs. quadruple 'rescue therapy' for the eradication of Helicobacter pylori resistant to metronidazole, Aliment Pharmacol Ther 16(7): 1277-1282.

Kang, J.M., Kim, N., Lee, D.H., Park, Y.S., Kim, Y.R., Kim, J.S. et al. (2007) Second-line treatment for Helicobacter pylori infection: 10-day moxifloxacin-based triple therapy versus 2 -week quadruple therapy, Helicobacter 12(6): 623-628.

Lamouliatte, H., Mégraud, F., Delchier, J.C., Bretagne, J.F., Courillon-Mallet, A., De Korwin, J.D. et al. (2003) Second-line treatment for failure to eradicate Helicobacter pylori: a randomized trial comparing four treatment strategies, Aliment Pharmacol Ther 18(8): 791-797.

Malfertheiner, P., Megraud, F., O’Morain, C., Bazzoli, F., El-Omar, E. et al. (2007) Current concepts in the management of Helicobacter pylori infection: the Maastricht III Consensus Report, Gut 56(6): 772-781.

Miehlke, S., Hansky, K., Schneider-Brachert, W., Kirsch, C., Morgner, A., Madisch, A. et al. (2006) Randomized trial of rifabutin-based triple therapy and high-dose dual therapy for rescue treatment of Helicobacter pylori resistant to both metronidazole and clarithromycin, Aliment Pharmacol Ther 24(2): 395-403.

Miehlke, S., Schneider-Brachert, W., Kirsch, C., Morgner, A., Madisch, A., Kuhlisch, E. et al. (2008) One-week once-daily triple therapy with esomeprazole, moxifloxacin, and rifabutin for eradication of persistent Helicobacter pylori resistant to both metronidazole and clarithromycin, Helicobacter 13(1): 69-74.

Navarro-Jarabo, J.M., Fernández, N., Sousa, F.L., Cabrera, E., Castro, M., Ramírez, L.M. et al. (2007) Efficacy of rifabutin-based triple therapy as secondline treatment to eradicate Helicobacter pylori infection, BMC Gastroenterol 25(7): 31.

Nista, E.C., Candelli, M., Cremonini, F., Cazzato, I.A., Di Caro, S., Gabrielli, M. et al. (2003) Levofloxacin-based triple therapy vs. quadruple therapy in second-line Helicobacter pylori treatment: a randomized trial, Aliment Pharmacol Ther 18(6): 627-633.

Parente, F., Cucino, C. and Bianchi Porro, G. (2003) Treatment options for patients with Helicobacter pylori infection resistant to one or more eradication attempts, Dig Liver Dis 35(8): 523-528.

Perna, F., Zullo, A., Ricci, C., Hassan, C., Morini, S. and Vaira, D. (2007) Levofloxacin-based triple therapy for Helicobacter pylori re-treatment: role of bacterial resistance, Dig Liver Dis 39(11): 1001-1005.
Perri, F., Festa, V., Clemente, R., Quitadamo, M. and Andriulli, A. (2000) Rifabutin-based 'rescue therapy' for Helicobacter pylori infected patients after failure of standard regimens, Aliment Pharmacol Ther 14(3): 311-316.

Perri, F., Festa, V., Clemente, R., Villani, M.R., Quitadamo, M., Caruso, N. et al. (2001) Randomized study of two 'rescue' therapies for Helicobacter pyloriinfected patients after failure of standard triple therapies Am F Gastroenterol 96(1): 58-62.

Perri, F., Festa, V., Merla, A., Barberani, F., Pilotto, A. and Andriulli, A. (2003) Randomized study of different 'second-line' therapies for Helicobacter pylori infection after failure of the standard 'Maastricht triple therapy', Aliment Pharmacol Ther 18(8): 815-820.

Realdi, G., Dore, M.P., Piana, A., Atzei, A., Carta, M., Cugia, L. et al. (1999) Pretreatment antibiotic resistance in Helicobacter pylori infection: results of three randomized controlled studies, Helicobacter 4(2): 106-112.

Saad, R.J., Schoenfeld, P., Kim, H.M. and Chey, W.D. (2006) Levofloxacin-based triple therapy versus bismuth-based quadruple therapy for persistent Helicobacter pylori infection: a meta-analysis, Am $\mathcal{F}$ Gastroenterol 101(3): 488-496.

Take, S., Mizuno, M., Ishiki, K., Nagahara, Y., Yoshida, T., Inaba, T. et al. (2003) Interleukin-1beta genetic polymorphism influences the effect of cytochrome P 2C19 genotype on the cure rate of 1-week triple therapy for Helicobacter pylori infection, $A m \mathcal{F}$ Gastroenterol 98(11): 2403-2408.

Togawa, J., Inamori, M., Fujisawa, N., Takahashi, H., Yoneda, M., Kawamura, H. et al. (2005) Efficacy of a triple therapy with rabeprazole, amoxicillin, and faropenem as second-line treatment after failure of initial Helicobacter pylori eradication therapy,

Hepatogastroenterology 52(62): 645-648.

Tong, J.L., Ran, Z.H., Shen, J., Zhang, C.X. and Xiao, S.D. (2007) Meta-analysis: the effect of supplementation with probiotics on eradication rates and adverse events during Helicobacter pylori eradication therapy, Aliment Pharmacol Ther 25(2): 155-168.

Treiber, G., Ammon, S., Malfertheiner, P. and Klotz, U. (2002) Impact of furazolidone-based quadruple therapy for eradication of Helicobacter pylori after previous treatment failures, Helicobacter 7(4): 225-231.

Tursi, A., Elisei, W., Brandimarte, G., Giorgetti, G.M., Modeo, M.E. and Aiello, F. (2007) Effect of lactoferrin supplementation on the effectiveness and tolerability of a 7-day quadruple therapy after failure of a first attempt to cure Helicobacter pylori infection, Med Sci Monit 13(4): CR187-190.

Uygun, A., Ozel, A.M., Yildiz, O., Aslan, M., Yesilova, Z., Erdil, A. et al. (2008) Comparison of three different second-line quadruple therapies including bismuth subcitrate in Turkish patients with non-ulcer dyspepsia who failed to eradicate 
Visit SAGE journals online http://tag.sagepub.com

@SAGEJOURNALS
Online
Helicobacter pylori with a 14-day standard first-line therapy $\mathcal{F}$ Gastroenterol Hepatol 23(1): 42-45.

Van der Poorten, D. and Katelaris, P.H. (2007) The effectiveness of rifabutin triple therapy for patients with difficult-to-eradicate Helicobacter pylori in clinical practice, Aliment Pharmacol Ther 26(11-12): 1537-1542.

Watanabe, Y., Aoyama, N., Shirasaka, D., Maekawa, S., Kuroda, K., Miki, I. et al. (2003) Levofloxacin based triple therapy as a second-line treatment after failure of Helicobacter pylori eradication with standard triple therapy, Dig Liver Dis 35(10): 711-715.

Wong, W.M., Gu, Q., Lam, S.K., Fung, F.M., Lai, K.C., Hu, W.H. et al. (2003) Randomized controlled study of rabeprazole, levofloxacin and rifabutin triple therapy vs. quadruple therapy as second-line treatment for Helicobacter pylori infection, Aliment Pharmacol Ther 17(4): 553-560.

Wong, W.M., Gu, Q., Chu, K.M., Yee, Y.K., Fung, F.M., Tong, T.S. et al. (2006) Lansoprazole, levofloxacin and amoxicillin triple therapy vs. quadruple therapy as second-line treatment of resistant Helicobacter pylori infection, Aliment Pharmacol Ther 23(3): 421-427.

Yee, Y.K., Cheung, T.K., Chu, K.M., Chan, C.K., Fung, J., Chan, P. et al. (2007) Clinical trial: levofloxacin-based quadruple therapy was inferior to traditional quadruple therapy in the treatment of resistant Helicobacter pylori infection, Aliment Pharmacol Ther 26(7): 1063-1067.

Zullo, A., Vaira, D., Vakil, N., Hassan, C., Gatta, L., Ricci, C. et al. (2003) High eradication rates of Helicobacter pylori with a new sequential treatment, Aliment Pharmacol Ther 17(5): 719-726. 